\title{
Applications of Symmetry Methods to the Theory of Plasma Physics
}

\author{
Giampaolo CICOGNA ${ }^{\dagger}$, Francesco CECCHERINI ${ }^{\ddagger}$ and Francesco PEGORARO \\ † Dip. di Fisica and INFN, Largo B. Pontecorvo 3, Ed. B-C, 56127-Pisa, Italy \\ E-mail: cicogna@df.unipi.it \\ ‡ Dip. di Fisica, INFM and CNISM, Largo B. Pontecorvo 3, Ed. B-C, 56127 - Pisa, Italy \\ E-mail: ceccherini@df.unipi.it,pegoraro@df.unipi.it
}

Received October 17, 2005, in final form January 20, 2006; Published online February 02, 2006

Original article is available at http://www.emis.de/journals/SIGMA/2006/Paper017/

\begin{abstract}
The theory of plasma physics offers a number of nontrivial examples of partial differential equations, which can be successfully treated with symmetry methods. We propose three different examples which may illustrate the reciprocal advantage of this "interaction" between plasma physics and symmetry techniques. The examples include, in particular, the complete symmetry analysis of system of two PDE's, with the determination of some conditional and partial symmetries, the construction of group-invariant solutions, and the symmetry classification of a nonlinear PDE.
\end{abstract}

Key words: Lie point symmetries; partial differential equations; plasma physics

2000 Mathematics Subject Classification: 35A30; 35Q60; 22E70

\section{Introduction}

The theory of plasma physics offers a number of highly nontrivial examples of partial differential equations, which can be successfully treated with symmetry methods. The three different examples we are going to consider may clearly emphasize the reciprocal advantage of this "interaction" between plasma physicists and symmetry theorists: for the latters, plasma theory offers the opportunity of testing their methods in concrete problems; for the formers, symmetry techniques provide new solutions to their equations.

Our first example actually deals with the Liouville equation. This equation admits well known properties and a rich literature is devoted to it; there are however various similar but not equivalent forms of this equation, as we shall briefly point out, which admit solutions with different properties, of course. After some comments on the general peculiarities of our equation and of its symmetry properties, we shall provide some new, physically relevant, solutions.

The second example deals with a system of two coupled PDE's. This system appears to be very rich in various and nontrivial symmetry properties. We shall provide the algebra of its exact Lie point symmetries and examine some properties of this (infinite dimensional) algebra. The system will be also compared with the properties of similar equations coming from magnetohydrodynamics. Next we shall study some invariant solutions of this system, and discuss their physical aspects. This system also admits some relevant "weak" (conditional and partial) symmetries, as we shall show, leading to other families of solutions. In addition, we shall discuss some peculiarities of the problem, considering, in particular, the effect of "truncating" the system (putting some physical parameters equal to zero) on its symmetry properties.

The final example is actually a "symmetry classification" problem. We will propose indeed a nonlinear PDE containing two arbitrary functions and examine how symmetry properties 
depend on the choice of these functions, also with the help of the notion of the equivalence group. The conclusion is that, apart from very special cases, only dilation exact symmetries are admitted by some well defined classes of functions. In addition, one nontrivial conditional symmetry is admitted, leading to a particularly simple reduced ODE, which can be easily reduced to quadratures.

In this paper we will be concerned only with Lie point symmetries, see $[1,2,3,4,5,6,7]$, under usual and standard definitions and assumptions, as stated e.g. in [2, 7]. For a different approach to symmetries of differential equations, using differential form methods, see the recent review paper [8].

For all details concerning plasma physics, we refer to $[9,10]$ and to the papers quoted therein.

\section{Plasma kinetic equilibria described by Liouville equation}

Under suitable approximations and in appropriate experimental conditions, the configuration of an isothermal plasma embedded in a stationary magnetic field is 2-dimensional (planar: say, in the plane $x, y$ ). It can be shown (see [10] and ref. therein) that such a configuration is completely described by a function $u=u(x, y)$, which is the component of the magnetic vector potential perpendicular to the $x, y$ plane, and which satisfies the classical "elliptic" Liouville equation

$$
\nabla^{2} u+\exp (2 u)=0
$$

Let us write, first of all, its most general 1-dimensional solution $u=u_{1}(x)$ which depends only on $x$ :

$$
u_{1}(x)=-\ln \left(\frac{1}{|c|} \cosh \left(c x+c^{\prime}\right)\right), \quad c, c^{\prime}=\text { const },
$$

with $c=1, c^{\prime}=0$, this is called in plasma theory the 1-dimensional Harris sheet pinch solution.

As is well known (see e.g. [11, 12, 13]), equation (1) admits the infinite dimensional algebra of Lie point symmetries generated by the vector fields

$$
X=\xi(x, y) \frac{\partial}{\partial x}+\eta(x, y) \frac{\partial}{\partial y}+\zeta(x, y) \frac{\partial}{\partial u},
$$

where $\xi(x, y), \eta(x, y)$ are arbitrary harmonic conjugated functions, and $\zeta(x, y)=-d \xi / d x$.

It can be remarked for the sake of completeness, that the "hyperbolic" Liouville equation, analogous to (1),

$$
\frac{\partial^{2} u}{\partial x^{2}}-\frac{\partial^{2} u}{\partial y^{2}}+\exp (2 u)=0
$$

admits similar but not identical symmetry properties: in particular, in the hyperbolic case the functions $\xi, \eta$ in (3) can be arbitrary functions (see [14, 15] and [6, Vol. 1, p. 204]).

Putting, in the case of equation (1),

$$
\phi=\phi(z)=\xi+i \eta \quad \text { with } \quad z=x+i y
$$

the function $\phi(z)$ is then an arbitrary holomorphic function of the complex variable $z=x+i y$, and the symmetry property stated above for equation (1) implies that, choosing such a function $\phi(z)$, one can construct continuous families of solutions starting from any given solution to $(1)$. 
On the other hand, it has been shown by Liouville $[13,16,17,18]$ that the most general solution to (1) can be written in the form

$$
u(x, y)=\ln \left(\frac{2\left|\gamma_{z}(z)\right|}{1+|\gamma(z)|^{2}}\right), \quad z=x+i y,
$$

where the "generating function" $\gamma=\gamma(z)$ is another arbitrary holomorphic function, and $\gamma_{z}(z)=$ $d \gamma / d z$. For instance, the choice $\gamma=\exp (z)$ produces the above mentioned 1-dimensional Harris solution. The slightly more general choice $\gamma(z)=k \exp (k z)+\kappa$, with $\kappa=\sqrt{k^{2}-1}, k \in \mathbb{R}$, $|k|>1$, gives the solution

$$
u(x, y)=-\ln (\cosh (k x)+(\kappa / k) \cos (k y))
$$

which describes a magnetic field configuration with a chain of magnetic "islands" analogous to "cat's eyes" vortex chain in hydrodynamics.

It can also be noted that, choosing $\gamma(z)=z^{a}(a>0)$ in (5), one obtains radial solutions to $(1)$

$$
u=-\ln \left[\frac{r}{2 a}\left(r^{a}+\frac{1}{r^{a}}\right)\right], \quad r^{2}=x^{2}+y^{2}
$$

which present a logarithmic singularity at the origin unless $a=1$. More precisely, it can be shown the following result $[18,19]$.

The most general solution to (1) which is globally defined and satisfies the "normalization condition"

$$
\int_{\mathbb{R}^{2}} \exp (2 u) d x d y<\infty
$$

is the solution generated by $\gamma(z)=c\left(z-z_{0}\right), c \in \mathbb{R}, c \neq 0$, i.e. a solution radially symmetric with respect to a point $\left(x_{0}, y_{0}\right) \in \mathbb{R}^{2}$, of the form (with $k=1 / c$ )

$$
u(x, y)=\ln \left(\frac{2|k|}{k^{2}+\left(x-x_{0}\right)^{2}+\left(y-y_{0}\right)^{2}}\right)
$$

which is known as the Bennet pinch solution.

Putting $\gamma(z)=\exp (\beta(z))$, one obtains from (5) this other useful expression of the general solution to (1)

$$
u(x, y)=-\ln \left(\frac{\cosh (\operatorname{Re} \beta(z))}{\left|\beta_{z}(z)\right|}\right) .
$$

This formula and the above equation (5) are the two expressions commonly used in the applications in plasma physics. It can be noted, incidentally, that if one considers the other equation

$$
\nabla^{2} u-\exp (2 u)=0
$$

(or $\nabla^{2} u+\exp (-2 u)=0$ ) slightly different from (1), several different representations are known of its general solutions [11, 13], but not all admit an analogous expression for our equation (1). For instance, equation (6) admits the two different solutions depending only on $x$

$$
u^{(1)}(x)=-\ln |\sinh x| \quad \text { and } \quad u^{(2)}(x)=-\ln |\sin x|
$$

whereas the solution given in (2) is the most general solution to (1) depending on $x$. One can also show that the elliptic Liouville equation in the form (6) does not possess solutions valid in the entire plane, while equation (1) does possess such solutions. 
For a full discussion about this (not marginal) point and a comparison between the possible expressions representing the various solutions of these Liouville equations, see [20]. It can be also noted that equation (6) has the same symmetry properties as equation (1).

Another peculiarity shared by all these types of Liouville equations is the possibility of studying and finding their solutions by means of Bäcklund transformations. Also in this approach, elliptic and hyperbolic equations actually behave a little bit differently: see e.g. [13, 21, 22]. We do not insist on this topic, which goes beyond the scope of the present paper.

Coming back to our equation (1), let us consider for instance the Lie symmetry determined by $\phi(z)=z^{2}$, i.e.

$$
X=\left(x^{2}-y^{2}\right) \frac{\partial}{\partial x}+2 x y \frac{\partial}{\partial y}-2 x \frac{\partial}{\partial u} .
$$

Starting from the Harris solution, we then obtain the following family of solutions, describing a continuously deformed family of curved plasma sheet configurations

$$
u(x, y)=-\ln \left[\left(1-2 \lambda x+\lambda^{2} r^{2}\right) \cosh \frac{x-\lambda r^{2}}{1-2 \lambda x+\lambda^{2} r^{2}}\right],
$$

where $r^{2}=x^{2}+y^{2}, \lambda \in \mathbb{R}$ is the group parameter. The generating function $\gamma$ is now

$$
\gamma=\gamma(z, \lambda)=\exp \left(\frac{z}{1-\lambda z}\right)
$$

in this way, the essential singularity of the generating function of Harris solution is shifted from infinity to $x=1 / \lambda$.

Even more in general, one can perform an arbitrary holomorphic transformation

$$
z \rightarrow \widetilde{z}=\psi(z)
$$

and consider then the new generating function $\widetilde{\gamma}(z) \equiv \gamma(\psi(z))$; thanks to (5), one obtains in this way other solutions to (1). Actually, it can be shown [11] that any solution to (1) can be obtained in this way; this means in particular that any solution can be transformed (locally) into the 1-dimensional solution (2) by means of a suitable holomorphic transformation $z \rightarrow \psi(z)$.

The close relationship between the generating function $\gamma(z)$ of any solution of (1) and symmetry properties, can be also emphasized by the following result [10].

Proposition 1. Let $u=u(x, y)$ be any solution to $(1), \gamma(z)$ its generating function and $X_{0}$ the Lie vector field which leaves invariant this solution: then

$$
\gamma_{z}(z) \phi_{0}(z)=i \gamma(z)
$$

where $\phi_{0}(z)$ is the holomorphic function which determines $X_{0}$.

For instance, for the above solution (2), one has $\gamma=\exp \left(c z+c^{\prime}\right)\left(\right.$ with $\left.c, c^{\prime} \in \mathbb{R}\right)$, and then from $(7) \phi_{0}=i / c$, i.e. $X_{0}=\partial / \partial y$, as expected.

To conclude this section, let us point out, among many other solutions, the three following examples, for their special interest to plasma physics (see [10]).

Choosing as generating function

$$
\gamma(z)=(1+p z)^{1 / p}, \quad p>0
$$

one obtains a one-parameter family of magnetic configurations with circular field lines, which includes the Bennet pinch for $p=1$, ring-like structures for $0<p<1$, and which gives the Harris solution at the limit $p \rightarrow 0$. Instead, the solution generated by

$$
\gamma(z)=p \operatorname{Erf}(z)
$$


produces a sort of bar-like structure in the plasma current distribution. Finally, starting from

$$
\gamma(z)=\frac{z+p}{z-p} \exp z
$$

one gets a solution which can be used to model some important features of the Earth magnetotail.

\section{A system of PDE's for magnetized plasmas}

The case we are now considering, which is the most elaborate and richest in different symmetry properties, is a system of two PDE's for the two functions $u=u(x, y, t), v=v(x, y, t)$.

The system is the following

$$
\begin{aligned}
& \frac{\partial}{\partial t}\left(u-\nabla^{2} u\right)-\left\{u-\nabla^{2} u, v\right\}+\left\{u, \nabla^{2} v\right\}=0, \\
& \frac{\partial}{\partial t}\left(\nabla^{2} v\right)-\left\{u, \nabla^{2} u\right\}+\left\{v, \nabla^{2} v\right\}=0,
\end{aligned}
$$

where the $\{\cdot, \cdot\}$ is defined by

$$
\{f, g\}=\frac{\partial f}{\partial x} \frac{\partial g}{\partial y}-\frac{\partial g}{\partial x} \frac{\partial f}{\partial y}
$$

or, written in a more "symmetric" and compact form,

$$
\frac{\partial}{\partial t}\left(u-\nabla^{2} u \pm \nabla^{2} v\right)+\left\{v \pm u, u-\nabla^{2} u \pm \nabla^{2} v\right\}=0 .
$$

In this system of PDE's the functions $u(x, y, t), v(x, y, t)$ describe respectively the time dependent magnetic and electric potentials of a 2-dimensional planar configuration of a fluid plasma, embedded in a strong magnetic field orthogonal to the $x, y$ plane and with a shear magnetic field in this plane.

\subsection{The Lie algebra of symmetries}

With the help of some appropriate computer package, e.g. [23, 24, 25], it is possible to show the following result:

Proposition 2. The system (8) admits the Lie algebra of symmetries generated by the following operators:

$$
\begin{aligned}
& X_{1}=\frac{\partial}{\partial t}, \quad X_{2}=y \frac{\partial}{\partial x}-x \frac{\partial}{\partial y}, \quad X_{3}=\frac{\partial}{\partial u}, \\
& X_{4}=-t y \frac{\partial}{\partial x}+t x \frac{\partial}{\partial y}+\frac{x^{2}+y^{2}}{2} \frac{\partial}{\partial v}
\end{aligned}
$$

and by the family of operators, depending on three arbitrary smooth functions $H(t), A(t), B(t)$ :

$$
\begin{aligned}
& X_{H}=H(t) \frac{\partial}{\partial v}, \\
& X_{(A, B)}=A(t) \frac{\partial}{\partial x}+B(t) \frac{\partial}{\partial y}+\left(x B_{t}-y A_{t}\right) \frac{\partial}{\partial v},
\end{aligned}
$$

where $A_{t}=d A / d t$, etc., which generate an infinite dimensional subalgebra (actually: an ideal). 
The nonvanishing commutation rules between the above defined symmetry generators are the following:

$$
\begin{aligned}
& {\left[X_{1}, X_{4}\right]=-X_{4}, \quad\left[X_{1}, X_{H}\right]=X_{H_{t}}, \quad\left[X_{1}, X_{(A, B)}\right]=X_{\left(A_{t}, B_{t}\right)},} \\
& {\left[X_{2}, X_{(A, B)}\right]=X_{(-B, A)}, \quad\left[X_{4}, X_{(A, B)}\right]=-X_{(-t B, t A)}, \quad\left[X_{(A, B)}, X_{(C, D)}\right]=X_{\widetilde{H}},}
\end{aligned}
$$

where $\widetilde{H}=A C_{t}-B D_{t}-C B_{t}+D A_{t}$.

The operators $X_{1}, X_{2}, X_{3}$ generate respectively time translation, space rotation and the translation $u \rightarrow u+k$, and together with the operator $X_{4}$ form a subalgebra of the algebra of the symmetries; the operators $X_{(A, B)}$ include in particular space translations $\partial / \partial x$ and $\partial / \partial y$. The operator $X_{H}$ generates the translation $v \rightarrow v+H(t)$; this operator and $X_{3}$ (which is the center of the algebra) do not change either the magnetic field or the plasma velocity. Some properties of the two remaining operators $X_{4}$ and $X_{(A, B)}$ will be considered in the next subsection.

\subsection{Symmetry properties and group-invariant solutions}

Before considering group-invariant solutions of system (8), let us look for an optimal system (see e.g. [2]) of 1-dimensional subalgebras of the algebra of symmetries listed in Proposition 2. It is easily seen that in the adjoint representation the action of the operators $X_{H}$ and $X_{(A, B)}$ on all the vector fields $X_{\alpha}$ (where $X_{\alpha}$ denotes any of the operators defined above) has the effect of transforming the functions $H(t), A(t), B(t)$ and does not touch the other operators; the same happens for the action of any $X_{\alpha}$ on $X_{H}$ and $X_{(A, B)}$. For instance, one has:

$$
\begin{aligned}
& \operatorname{Ad}\left(\exp \left(\lambda X_{(A, B)}\right)\right) \frac{\partial}{\partial t}=\frac{\partial}{\partial t}+\lambda X_{\left(A_{t}, B_{t}\right)}-\frac{\lambda^{2}}{2}\left(A A_{t t}-B B_{t t}\right) \frac{\partial}{\partial v} \\
& \operatorname{Ad}\left(\exp \left(\lambda X_{(A, B)}\right)\right) X_{(C, D)}=X_{(A, B)}-\lambda\left(A C_{t}-B D_{t}-C B_{t}+D A_{t}\right) \frac{\partial}{\partial v} .
\end{aligned}
$$

In view of the physical applications, it is more convenient to consider the functions $H, A, B$ as arbitrary functions, and therefore we do not include in the classification the operators $X_{H}$, $X_{(A, B)}$. Restricting to the operators $X_{1}, X_{2}$ and $X_{4}$ (notice that $X_{3}$ acts trivially on all the $X_{\alpha}$ ), an optimal system is

$$
X_{1}=\frac{\partial}{\partial t}, \quad X_{2}=y \frac{\partial}{\partial x}-x \frac{\partial}{\partial y}, \quad X_{a}=a \frac{\partial}{\partial t}+X_{4},
$$

where $a$ is a real parameter.

The equations for time-invariant or rotationally-invariant solutions can be easily deduced; on the other hand, they are actually very special solutions, of no general interest. The same is true for solutions invariant under $X_{H}$ (i.e. solutions with $v \equiv 0$ ). We then consider in some detail the symmetries $X_{4}$ and $X_{(A, B)}$. It is convenient to examine first the symmetry $X_{(A, B)}$.

1 ) The symmetry $X_{(A, B)}$ expresses the property that, if $u(x, y, t), v(x, y, t)$ is a solution of (8), then also $^{1}$

$$
\begin{aligned}
& \widetilde{u}(x, y, t):=u(x-A(t), y-B(t), t), \\
& \widetilde{v}(x, y, t):=x B_{t}-y A_{t}-\frac{1}{2}\left(A B_{t}-A_{t} B\right)+v(x-A(t), y-B(t), t)
\end{aligned}
$$

solve our system (8), for any $A(t), B(t)$. It must be noticed that this corresponds to a change of spatial coordinates into a moving frame which produces in turns the additional term $x B_{t}-$

\footnotetext{
${ }^{1}$ The parameter $\lambda$ which should be introduced to parametrize this family of solutions can be clearly absorbed in the (arbitrary) functions $A$ and $B$.
} 
$y A_{t}-(1 / 2)\left(A B_{t}-A_{t} B\right)$ in the component $v$. Since $v$ is proportional to the electric potential, we can interpret this symmetry as expressing the fact that a time dependent, spatially uniform, electric field imposed on the system induces a uniform time-dependent electric drift.

We want now to look for the solutions to (8) which are invariant under $X_{(A, B)}$. Let us recall that if a system of PDE's for the $q$ functions $u_{a}=u_{a}(x)$ of the $p$ variables $x_{i}$ admits a symmetry $X=\xi_{i} \partial / \partial x_{i}+\zeta_{a} \partial / \partial u_{a}, i=1, \ldots, p, a=1, \ldots, q$, then the $X$-invariant solutions must satisfy the $q$ equations

$$
\zeta_{a}-\xi_{i} \frac{\partial u_{a}}{\partial x_{i}}=0
$$

These equations in our case take the form

$$
A u_{x}+B u_{y}=0, \quad A v_{x}+B v_{y}=x B_{t}-y A_{t} .
$$

The first equation can be interpreted as the requirement that the displacement produced by the electric drift is parallel to the field lines of the shear magnetic field, while the second expresses the requirement that the electric potential of the displaced plasma element remains constant.

Instead of looking directly for the invariant solutions to (8) starting from (14), it can be more interesting to give preliminarily the expression of equations (8) when written in the "canonical coordinates" (or "symmetry-adapted coordinates") [2, 7]. In our case these coordinates can be chosen as follows

$$
s=B(t) x-A(t) y, \quad w=\frac{A(t) x+B(t) y}{A^{2}(t)+B^{2}(t)},
$$

where $s$ is (together with $t$ ) an invariant coordinate under the symmetry $X_{(A, B)}$, and $w$ is the coordinate "along the action" of the symmetry, i.e. $X_{(A, B)} w=1$. Notice also that $\{f, g\}_{x, y}=$ $\{f, g\}_{s, w}$.

Putting, as indicated by (14),

$$
u=U(s, w, t), \quad v=Q(x, y, t)+V(s, w, t)
$$

where $Q$ is a quadrupolar term given by

$$
Q=\frac{1 / 2}{A^{2}+B^{2}}\left(\left(A_{t} B+A B_{t}\right)\left(x^{2}-y^{2}\right)-2 x y\left(A A_{t}-B B_{t}\right)\right),
$$

we obtain from (8) the following system

$$
\begin{gathered}
\frac{\partial}{\partial t}\left(U-\widetilde{\nabla}^{2} U \pm \widetilde{\nabla}^{2} V\right)+\left\{V \pm U, U-\widetilde{\nabla}^{2} U \pm \widetilde{\nabla}^{2} V\right\} \\
+\frac{2 s}{\left(A^{2}+B^{2}\right)^{2}} \frac{\partial}{\partial w}\left(U-\widetilde{\nabla}^{2} U \pm \widetilde{\nabla}^{2} V\right)=0,
\end{gathered}
$$

where $\widetilde{\nabla}^{2}=\left(A^{2}+B^{2}\right) \partial^{2} / \partial s^{2}+\left(A^{2}+B^{2}\right)^{-1} \partial^{2} / \partial w^{2}$. Here the different role of the two variables $s$ and $w$ is evident: in particular - as expected - there is no explicit dependence on $w$, whereas the last term, proportional to $s$, can be interpreted as a Coriolis-type contribution.

Now, if one looks for invariant solutions, i.e. for solutions of the form

$$
u=U_{0}(s, t), \quad v=Q(x, y, t)+V_{0}(s, t)
$$

the above equations (16) become

$$
\frac{\partial}{\partial t}\left(U_{0}-\left(A^{2}+B^{2}\right) U_{0, s s}\right)=0, \quad \frac{\partial}{\partial t}\left(\left(A^{2}+B^{2}\right) V_{0, s s}\right)=0 .
$$


These clearly imply

$$
U_{0}-\left(A^{2}+B^{2}\right) U_{0, s s}=F(s), \quad\left(A^{2}+B^{2}\right) V_{0, s s}=G(s),
$$

where $F(s), G(s)$ are arbitrary functions. Notice that $\partial^{2} / \partial x^{2}+\partial^{2} / \partial y^{2}=\left(A^{2}+B^{2}\right) \partial^{2} / \partial s^{2}$ and that these equations are actually ODE's, indeed the variable $t$ here appears merely as a parameter.

Special physically relevant solutions of this reduced system (and of the system (8), of course) can be easily obtained starting from particular choices of the arbitrary functions $A(t), B(t)$, $F(s), G(s)$ introduced above (see [9] for some explicit examples).

2) We now consider the symmetry $X_{4}$ of our system (8): it implies that if $u(x, y, t), v(x, y, t)$ is a solution, then also

$$
\begin{aligned}
& \widetilde{u}(x, y, t):=u(x \cos (\lambda t)+y \sin (\lambda t),-x \sin (\lambda t)+y \cos (\lambda t), t), \\
& \widetilde{v}(x, y, t):=v(x \cos (\lambda t)+y \sin (\lambda t),-x \sin (\lambda t)+y \cos (\lambda t), t)+\lambda \frac{x^{2}+y^{2}}{2}
\end{aligned}
$$

is a family of solutions to (8) for any $\lambda \in \mathbb{R}$. This represents a sort of rotated solutions with angular velocity $\lambda$ plus a radial term in the component $v$ which gives the additional velocity field corresponding to the rotation.

The solutions to (8), which are invariant under symmetry (10), must satisfy the invariance condition

$$
y \frac{\partial u}{\partial x}-x \frac{\partial u}{\partial y}=0, \quad y \frac{\partial v}{\partial x}-x \frac{\partial v}{\partial y}=-\frac{r^{2}}{2 t},
$$

where $r^{2}=x^{2}+y^{2}$. It is easy to find that they are of the form, with $\theta=\arccos (x / r)$,

$$
u=U^{(0)}(r, t), \quad v=\frac{r^{2}}{2 t} \theta+V^{(0)}(r, t),
$$

where $U^{(0)}(r, t), V^{(0)}(r, t)$ satisfy the linear and uncoupled homogeneous PDE's

$$
\begin{aligned}
& r^{2} U_{r r r}^{(0)}-2 r t U_{r r t}^{(0)}+r U_{r r}^{(0)}-2 t U_{r t}^{(0)}-r^{2} U_{r}^{(0)}+2 r t U_{t}^{(0)}+3 U_{r}^{(0)}=0, \\
& 2 r t V_{r r t}^{(0)}-r^{2} V_{r r r}^{(0)}+2 t V_{r t}^{(0)}-r V_{r r}^{(0)}+5 V_{r}^{(0)}=0 .
\end{aligned}
$$

A simple solution of the first equation is, e.g., $U^{(0)}=r^{2} t$, whereas the second one admits solutions of the form $V^{(0)}=r^{a} t^{b}$ with $b=\left(a^{2}-2 a-4\right) / 2 a$, for any $a$. It can be noted in particular that the special form of the component $v$ in (17), which has a fixed term containing a "cut" discontinuity, looks as a spiral and expresses the fact that this solution contains a $\theta$ independent azimuthal electric field, vanishing for $t \rightarrow \pm \infty$. Solutions of this form are not new in plasma theory and in magnetohydrodynamics: see e.g. [26] and [6, Vol. 1, p. 393]. See also Subsection 3.4 for other remarks and comments on various related physical aspects.

It is remarkable that, in both cases of symmetries (12) and (10), the invariant solution $u$ satisfies a linear equation (therefore linear superposition principle holds), and that the same is true for $v$, apart from a fixed additional term (for some comment on this point we refer to [9]).

3) Finally, one can also look for solutions invariant under symmetries of the form, e.g.,

$$
\frac{\partial}{\partial t}+X_{H}, \quad \frac{\partial}{\partial t}+X_{(A, B)}
$$

(or similar combinations: see our remark on the optimal system of symmetries at the beginning of this section). Clearly, the most convenient choice of symmetry to be considered, as well as 
the choice of the arbitrary functions $H, A, B$ involved, can be suggested by the specific case to be examined or by the experimental configuration. Just to give some examples, let us consider the two symmetries (18).

For the first symmetry, it can be easily seen that the invariant solutions have the form

$$
u=U(x, y), \quad v=T(t)+V(x, y),
$$

where $T_{t}=H(t)$ and $U, V$ satisfy the system

$$
\left\{U-\nabla^{2} U, V\right\}=\left\{U, \nabla^{2} V\right\}, \quad\left\{U, \nabla^{2} U\right\}=\left\{V, \nabla^{2} V\right\} .
$$

Considering instead the symmetry $\partial / \partial t+X_{(A, B)}$, one finds that the invariant solutions have the form

$$
\begin{aligned}
& u=U(x-\alpha(t), y-\beta(t)), \\
& v=(x-\alpha(t)) B-(y-\beta(t)) A+\alpha B-\beta A+V((x-\alpha(t), y-\beta(t)),
\end{aligned}
$$

where $\alpha_{t}=A, \beta_{t}=B$, and $U, V$ must satisfy a system of nonlinear PDE's.

\section{3 "Weak" symmetries}

The above system (8) admits also several interesting "non-exact" (here generically called "weak") symmetries; we are going to consider an example of conditional symmetry (see [27, 28, 29, 30, $31,32]$ ), and one of partial symmetry (according to the definition given in [33], see also [34] and below).

As well known, any vector field $X=\xi_{i} \partial / \partial x_{i}+\zeta_{a} \partial / \partial u_{a}$ is a conditional symmetry for a differential equation (or a system thereof) $\Delta=0$ if the system $\Delta=0$ enlarged with the invariance condition (13) admits some solution. If this is the case, a conditional symmetry allows the reduction of the initial equation into a reduced form, and in this way one can obtain other invariant solutions: see [27, 28, 29, 30, 31, 32]. See also [35, 36, 37, 38, 39, 40, 41, 42, 43] for careful discussions about various related problems and reduction procedures.

An example of conditional symmetry leading to interesting solutions to (8) is the following (more precisely, it is a "contact conditional symmetry"):

$$
X=u_{y} \frac{\partial}{\partial u}+v_{x} \frac{\partial}{\partial v} .
$$

From this symmetry, one finds e.g. solutions to (8) of this form

$$
u=\sin [k(x-T(t))], \quad v=\exp ( \pm \kappa y)-T_{t}(t) y,
$$

where $k, \kappa \in \mathbb{R}$ with $k^{2}-\kappa^{2}=1$ and $T(t)$ is arbitrary, or similar solutions where the functions sin and exp are exchanged. Solutions of this form are interesting due to the presence of terms depending on $x-T(t)$ describing generalized wave propagation.

It is known that there are some delicate points related to the definition of conditional symmetries: see $[35,36,37,38,39,40,41,42]$; this is actually related to the introduction of a subtler classification of the notion of conditional symmetry [44, 45]. We do not deal here with this problem, and we prefer to consider a particularly interesting example of partial symmetry for our system (8).

While exact symmetries transform any solution of the given problem into another solution, conditional symmetries do not transform - in general - solutions into solutions, as well known. Partial symmetries play in a sense an intermediate role, indeed they transform solutions belonging to a proper subset of solutions into solutions in the same subset. This subset is defined 
in the following way. Let us assume that some vector field $X$ is not an exact symmetry for a differential equation (or a system) $\Delta=0$ : this means that, denoting by $X^{*}$ the appropriate prolongation of $X$, one has $\left.X^{*}(\Delta)\right|_{\Delta=0} \neq 0$. Let us then introduce the condition

$$
\Delta^{(1)}:=X^{*}(\Delta)=0
$$

as a new equation, and consider the enlarged system

$$
\Delta=\Delta^{(1)}=0 .
$$

It is clear that if $X$ is an exact symmetry of this enlarged system, then the subset $\mathcal{S}^{(1)}$ of the simultaneous solutions of this system (if not empty, of course) is a "symmetric set of solutions" to $\Delta=0$, i.e. a proper subset of solutions which have the property of being mapped the one into another by the vector field $X$. It is also clear that this property is not shared by the other solutions to $\Delta=0$ not belonging to the subset $\mathcal{S}^{(1)}$. In principle, this procedure can be iterated, see $[33,34]$, but no iteration is necessary in the example we are considering.

The vector field we want to deal with is

$$
X=u \frac{\partial}{\partial u} \text {. }
$$

First of all, notice that this is trivially a conditional symmetry for (8), indeed the invariance condition simply amounts in this case to look for the special solutions to (8) with $u=0$, i.e. to the hydrodynamic limit. More interestingly, we now show that the vector field (21) is a nontrivial partial symmetry: notice that considering this vector field as a partial symmetry corresponds to looking for solutions to (8) with the property that the component $u$ admits a scaling property, i.e. for solutions $u, v$ such that also $\lambda u, v$ solve (8) for $\lambda \in \mathbb{R}$. Applying the prolongation $X^{*}$ to the system (8), and combining the resulting equation $\Delta^{(1)}=X^{*}(\Delta)$ with (8), one gets the new condition

$$
\left\{u, \nabla^{2} u\right\}=0,
$$

which is then the condition characterizing the subset $\mathcal{S}^{(1)}$ of solutions with the above specified property. It is easy to verify that the system of the three equations (8) and (22) is symmetric under (21), showing that (21) is indeed a partial symmetry for (8). Thanks to (22), we deduce this equation for $v$

$$
\frac{\partial}{\partial t}\left(\nabla^{2} v\right)+\left\{v, \nabla^{2} v\right\}=0
$$

which does not contain $u$, and is equivalent to the two-dimensional Euler equation for an incompressible fluid. On the other hand, the equation for $u$ is linear in $u$ : obviously, this agrees with the presence of the (partial) symmetry given by (21).

Before considering examples of particular simple solutions, let us come back to an important physical feature of our initial system of differential equations (8). This system indeed would actually contain some physical parameters that we have normalized to the unity up to now. There are physical situations, however, where one of these parameters turns out to be negligible and can be put equal to zero with a good approximation [9]: this coefficient multiplies the term $\left\{u, \nabla^{2} v\right\}$ in (8). For this reason, it can be interesting to repeat calculations determining symmetry properties and solutions of the approximate system. The quite surprising result is summarized in the following

Proposition 3. The truncated system

$$
\frac{\partial}{\partial t}\left(u-\nabla^{2} u\right)-\left\{u-\nabla^{2} u, v\right\}=0, \quad \frac{\partial}{\partial t}\left(\nabla^{2} v\right)-\left\{u, \nabla^{2} u\right\}+\left\{v, \nabla^{2} v\right\}=0
$$


admits precisely the same exact symmetries as the original one (8). The same is also true if one or both of the other equations

$$
\left\{u, \nabla^{2} v\right\}=0, \quad\left\{u, \nabla^{2} u\right\}=0
$$

are appended to the above system (therefore, even if the partial symmetry (21) is taken in consideration also within this approximation).

Now observing that condition (22) implies $\nabla^{2} u=A(u, t)$ for some smooth function $A$, and similarly $\left\{u, \nabla^{2} v\right\}=0$ implies $\nabla^{2} v=B(u, t)$, the system (23)-(24) becomes

$$
\begin{aligned}
& \left(1-A_{u}\right)\left(u_{t}-\{u, v\}\right)=A_{t}, \quad \nabla^{2} u=A(u, t), \\
& -B_{u}\left(u_{t}-\{u, v\}\right)=B_{t}, \quad \nabla^{2} v=B(u, t) .
\end{aligned}
$$

Therefore, assuming e.g. $A_{u}=1$ forces $A_{t}=0$; instead if $A_{u} \neq 1, B_{u} \neq 0$, but $A_{t}=B_{t}=0$, the above system takes the very simple form

$$
u_{t}=\{u, v\}, \quad \nabla^{2} u=A(u), \quad \nabla^{2} v=B(u) .
$$

Elementary solutions of this system (which are also solutions to (8), of course) can be immediately found, for instance, just to give some simple examples,

$$
\left.u=c_{1} \sin (k(x-t))+c_{2} \sin (k(y-t))+c_{3}\right), \quad v=x-y,
$$

where $c_{i}, k$ are arbitrary constants, or also $u=2 t-\theta, v=x^{2}+y^{2}$, with $\theta=\arctan (y / x)$, and $u=U(y-t), v=x$, where $U$ is an arbitrary regular function.

\subsection{General remarks}

As a general remark about the problem examined in this section let us point out that the set of equations (8) are of the interest for the study of the nonlinear dynamics of fluid plasma configurations and in particular for the interaction of magnetized plasma vortices and for the development of collisionless magnetic field line reconnection. In particular, the explicit solutions found in the present article using Lie point symmetries have a direct physical interpretation; for instance, the solutions (15)-(16) have a role in the recent investigation of nonlinear evolution and saturation of magnetic reconnection instabilities $[46,47]$ as plasma configurations forced from the boundaries. We note that equations (8) obey boundary conditions that are determined by values of the electric and magnetic fields at the boundaries of the domain where the plasma is enclosed. These boundary conditions may include plasma fluxes through the boundaries. Referring for example to the solutions (15)-(16), we see that they correspond to a plasma velocity pattern controlled by the boundary conditions that determine the arbitrary functions $A(t)$ and $B(t)$. The required boundary conditions correspond to imposing the electric field at these plates: the tangential component is proportional to $\nabla v$ and determines the velocity pattern, while the normal component is proportional to $\partial u / \partial t$ and determines the magnetic terms.

It is interesting to observe that the system (8) can be seen as a generalization of the twodimensional limit (i.e., the case of solutions independent of the coordinate $z$ orthogonal to the plane $(x, y)$ ), of the so-called Reduced Magnetohydrodynamic equations (Kadomtsev-Pogutse equations) [48], see also [11]. The symmetry properties of these equations, that are frequently used to describe plasmas embedded in a very strong magnetic field, have been studied in [26], see also [6, Vol. 2, p. 390]. It can be remarked that a common feature of the vector fields describing the symmetry properties of all these equations is the presence in their expression of one or more arbitrary functions (see also [49]) constant along magnetic (and velocity) field 
lines. The symmetry properties of the three dimensional visco-resistive magnetohydrodynamic equations in the incompressible limit have been studied in [50], see also [6, Vol. 1, p. 389].

Finally, the study of the partial symmetry (21) has been useful not only to obtain a reduction of the initial equations, leading also to new particular solutions, but also to point out a special property of the system (8): it can be suitably truncated as in equation (23) (i.e. a physical parameter can be put equal to zero) without altering its general symmetry properties.

\section{Symmetry classification of an equation for axisymmetric plasma configurations}

Our third example deals with a completely different situation. The equation we are going to examine contains indeed two arbitrary functions $F(u)$ and $G(u)$ of the unknown variable $u=u(x, y)$, and the goal is now to perform the symmetry classification of this equation, i.e. to find those $F, G$ for which the equation admits nontrivial symmetries. In general, the symmetry properties of an equation may strongly depend on the choice of the arbitrary functions involved.

Just to recall briefly a significant example, let us point out the case of the nonlinear Laplace equation $\nabla^{2} u=F(u)$, with $u=u(x, y)$ (or its hyperbolic counterpart, i.e. the nonlinear wave equation $u_{x x}-u_{y y}=F(u)$ ). Symmetry properties of equations of this form have been exensively studied; a complete symmetry classification of the nonlinear wave equations can be found in [14]. Summarizing the results, one has that these equations, excluding for a moment the particular cases $F=u$ and $F=1$, admit the infinite dimensional algebra of symmetries already mentioned (Section 2) if $F(u)=\exp (u)$ (the Liouville case), the nearly trivial symmetry

$$
X=(k-1)\left(x \frac{\partial}{\partial x}+y \frac{\partial}{\partial y}\right)-2 u \frac{\partial}{\partial u}
$$

if $F(u)=u^{k}$, and only the obvious symmetries (translations and rotations of the variables $x, y$ ) otherwise. In the case $F=u$ one finds the standard symmetries of the linear equations; finally, if $F=1$, the infinite-dimensional algebra of symmetries given by (3) is replaced by

$$
X=\xi(x, y) \frac{\partial}{\partial x}+\eta(x, y) \frac{\partial}{\partial y}+(c u+A(x, y)) \frac{\partial}{\partial u},
$$

where $c=$ const and $A(x, y)$ must satisfy

$$
\nabla^{2} A-2 \xi_{x}+c=0 .
$$

Performing the symmetry classification of a given equation may be not an easy task: for some explicit examples and the presentation of the techniques used in each situation, see e.g. [51, 52, $53,54,55]$ and the papers quoted therein.

The PDE we want to consider is

$$
u_{x x}+\frac{a}{x} u_{x}+u_{y y}=x^{2 p} F(u)+G(u),
$$

where $a, p$ are constants. With $a=-1, p=1$ this equation is known in plasma physics as the Grad-Schlüter-Shafranov equation (see [56]) and describes the magnetohydrodynamic force balance in a magnetically confined toroidal plasma. In this context, $u$ is the so-called magnetic flux variable, $x$ is a radial variable, then $x \geq 0$, while the two arbitrary flux functions $F(u)$, $G(u)$ are related to the plasma pressure and current density profiles.

According to the standard definitions and procedure (see [1]), we can now look for the equivalence group; preliminarily, we look for the kernel of the full groups of equation (26), i.e. the 
intersection of all groups admitted by (26) for any arbitrary choice of $F$ and $G$. It turns out that this kernel is almost trivial: it contains indeed only the translation of the variable $y$.

The complete symmetry analysis of the above equation (26) is a little bit tedious, but some simplification is provided for equations of this type, where the nonlinearity is present only in the arbitrary functions $F, G$. In this case indeed one can show that the coefficients $\xi, \eta, \zeta$ of the admitted symmetries $X=\xi \partial / \partial x+\eta \partial / \partial y+\zeta \partial / \partial u$ are subjected to some precise linear constraints, which admit a nice geometrical interpretation [57].

In the case of our equation (26), one obtains that, apart from the translation of the variable $y$, the equivalence group contains the following transformations:

i) the translation of the dependent variable $u$, i.e. $u \rightarrow u+k$,

ii) the scaling

$$
u \rightarrow \alpha u, \quad F \rightarrow \alpha F, \quad G \rightarrow \alpha G,
$$

iii) the scaling

$$
x \rightarrow \alpha x, \quad y \rightarrow \alpha y, \quad F \rightarrow \alpha^{-2(p+1)} F, \quad G \rightarrow \alpha^{-2} G .
$$

The conclusion can be summarized as follows.

Proposition 4. Apart from the particular cases where both $F$ and $G$ have the form $c_{1}+c_{2} u$, to be examined later, the only choices for the functions $F, G$ which lead to an equation admitting some symmetry (up to transition to equivalent functions via equivalence group) are the following:

$$
\text { a) } \quad F(u)=u^{1+(p+1) / q}, \quad G(u)=u^{1+1 / q}
$$

for all $q \neq 0$, where the admitted symmetry is

$$
X=x \frac{\partial}{\partial x}+y \frac{\partial}{\partial y}-2 q u \frac{\partial}{\partial u}
$$

and

$$
\text { b) } \quad F(u)=\exp ((1+p) u), \quad G(u)=\exp (u)
$$

with symmetry

$$
X=x \frac{\partial}{\partial x}+y \frac{\partial}{\partial y}-2 \frac{\partial}{\partial u} .
$$

In addition, one has that

c) if in equation (26) $p=-1$ and $G(u) \equiv 0$, then the scaling of the variables $x, y$, generated by

$$
X=x \frac{\partial}{\partial x}+y \frac{\partial}{\partial y}
$$

is a symmetry for all $F(u)$.

For instance, with account of the equivalence group transformations, the most general choice of functions in class $a$ ), is

$$
\left.a^{\prime}\right) \quad F(u)=c_{1}(c+u)^{1+(p+1) / q}, \quad G(u)=c_{2}(c+u)^{1+(1 / q)},
$$

where $c, c_{1}, c_{2}$ are constants, with symmetry

$$
X=x \frac{\partial}{\partial x}+y \frac{\partial}{\partial y}-2 q(c+u) \frac{\partial}{\partial u} .
$$


No new symmetry appears with particular choices of the parameters $a$ and $p$, and of the functions $F, G$ as well, apart from the following almost obvious exceptions. First of all, we exclude from our classification the case $a=0$ and $p=0$ (or $a=0$ and $F(u) \equiv 0$ ) because in this case our equation coincides with the already mentioned and well known nonlinear Laplace equation. Other possibilities appear if both $F$ and $G$ have the form $c_{1}+c_{2} u$. If $F=G=u$, the equation exhibits the standard symmetries of any linear equation. If $F=1, G=u$, there is a new admitted symmetry which can be written in the following way:

$$
X=(u+\Phi(x, y)) \frac{\partial}{\partial u},
$$

where, with $\mathcal{E}(u)$ denoting the left hand side of equation $(26), \Phi$ solves the equation

$$
\mathcal{E}(\Phi)=\Phi-x^{2 p}
$$

If $F=u, G=1$, the new symmetry has the same form as (28), but $\Phi$ must solve

$$
\mathcal{E}(\Phi)=x^{2 p} \Phi-1 \text {. }
$$

Thanks to the equivalence group of our equation, it remains only to consider the case $F=G=1$. In this case, although of no great interest in the applications, the situation is much more complicated. Indeed, the most general vector field $X$ belonging to the algebra of the admitted symmetries can be globally described in the following way:

$$
X=\left(c_{1} x+2 c_{2} x y\right) \frac{\partial}{\partial x}+\left(c_{0}+c_{1} y-c_{2}\left(x^{2}-y^{2}\right)\right) \frac{\partial}{\partial y}+\left(\left(c_{3}-c_{2} a y\right) u+\Psi(x, y)\right) \frac{\partial}{\partial u},
$$

where the function $\Psi(x, y)$ satisfies the equation

$$
\mathcal{E}(\Psi)+c_{3}-2 c_{1}+x^{2 p}\left(c_{3}-2 c_{1}(p+1)\right)-c_{2} y(4+a)-c_{2} x^{2 p} y(4(p+1)+a)=0 .
$$

There is, in addition, an interesting conditional symmetry for equation (26) in the case $a=-p \neq 0$ (which covers in particular the concrete case $a=-p=-1$ of the Grad-SchlüterShafranov equation), and $G(u)=k^{2} F(u)$ ( $k=$ const). The conditional symmetry is given by

$$
X=k \frac{\partial}{\partial x}+x^{p} \frac{\partial}{\partial y} .
$$

This symmetry is particularly interesting and useful because, with introduction of the symmetryinvariant variable

$$
s=\frac{1}{p+1} x^{p+1}-k y \quad \text { if } \quad p \neq-1, \quad \text { or } \quad s=\ln x-k y \quad \text { if } p=-1
$$

the PDE is transformed into the very simple reduced ODE

$$
u_{s s}=F(u)
$$

which can be easily integrated.

The class $(a)$ (or $\left(a^{\prime}\right)$ ) symmetry in Proposition 4 corresponds, for $a=-p=-1$, i.e. for the Grad-Schlüter-Shafranov equation, to spatial dilations where $u$ scales as $x^{-2 q}, y^{-2 q}$. An elementary solution obeying such a symmetry is obtained by taking, e.g., $q=-2$ and by considering "cylindrical" solutions with $\partial u / \partial y=0$. Choosing $F(u)=c_{1}$ and $G(u)=\left(8-c_{1}\right) u^{1 / 2}$, according to equations (27), we obtain from (26) $u=x^{4}$. By recalling the physical meaning of the functions $F(u)=c_{1}$ and $G(u)=\left(8-c_{1}\right) u^{1 / 2}$ we find that this solution describes a plasma configuration in the (dilation dependent) domain $0<x<x_{0}$ with a pressure $p$ of the form $p=$ 
$\left(c_{1} / 4 \pi\right)\left(x_{0}^{4}-x^{4}\right)$ and a total current $I$ along the cylinder of the form $I^{2}=\left[4\left(8-c_{1}\right) / 3\right]\left(x_{0}^{6}-x^{6}\right)$, where $\left[4\left(8-c_{1}\right) / 3\right]^{1 / 2} x_{0}^{3}$ is the current flowing along a conductor at $x=0$. The boundary conditions have been chosen such that the pressure and the total current vanish at $x=x_{0}$.

Finally we remark that the Grad-Schlüter-Shafranov equation is a special example of a family of equations that are used in plasma physics and in space physics and astrophysics [58] in order to describe time independent configurations of a plasma embedded in a magnetic field in the presence of plasma fluid motions (not included in the Grad-Schlüter-Shafranov equation). Similarly to the Grad-Schlüter-Shafranov equation, these equations depend on a set of arbitrary functions, but can change from elliptic to hyperbolic type depending on the (local) value of the plasma velocity.

\section{Conclusion}

In conclusion, we believe that the three examples considered in this paper may give a detailed idea of the opportunities offered by symmetry methods to the study of differential equations arising from modern physical theories. Let us emphasize in particular the completely different peculiarities of our examples, all suggested by plasma physics, and correspondingly the different role played by symmetry techniques in their application to each situation. For other different applications to plasma physics of similar methods and procedures, based on general symmetry properties, we refer also, e.g., to the papers [59, 60, 61, 62].

\section{Acknowledgements}

We are indebted to the referees for their useful comments and valuable suggestions. We also thank Nino Valenti for his precious help with a search in the literature.

[1] Ovsjannikov L.V., Group properties of differential equations, Novosibirsk, Izdat. Sibirsk. Otdel. Akad. Nauk SSSR, 1962 (English transl.: Group analysis of differential equations, New York, Academic Press, 1982).

[2] Olver P.J., Application of Lie groups to differential equations, 2nd ed., Berlin, Springer, 1988.

[3] Stephani H., Differential equations. Their solution using symmetries, Cambridge, University Press, 1989.

[4] Bluman G.W., Kumei S., Symmetries and differential equations, Berlin, Springer, 1989.

[5] Gaeta G., Nonlinear symmetries and nonlinear equations, Dordrecht, Kluwer, 1994.

[6] Ibragimov N.H. (Editor), CRC Handbook of Lie group analysis of differential equations, Boca Raton, CRC Press, 1994, Vol. 1; 1995, Vol. 2; 1996, Vol. 3.

[7] Bluman G.W., Anco S.C., Symmetry and integration methods for differential equations, New York, Springer, 2002.

[8] Harrison B.K., The differential form method for finding symmetries, SIGMA, 2005, V.1, paper 001, 12 pages; math-ph/0510068.

[9] Ceccherini F., Cicogna G., Pegoraro F., Symmetry properties of a system of Euler-type equations for magnetized plasmas, J. Phys. A: Math. Gen., 2005, V.38, 4597-4610.

[10] Ceccherini F., Montagna C., Pegoraro F., Cicogna G., Two-dimensional Harris-Liouville plasma kinetic equilibria, Phys. Plasmas, 2005, V.12, 052506-1/8.

[11] Gusyatnikova V.N., Samokhin A.V., Titov V.S., Vinogradov A.M., Yamaguzhin V.A., Symmetries and conservation laws of Kadomtsev-Pogutse equations, Acta Appl. Math., 1989, V.15, 23-64.

[12] Ibragimov N.H., Elementary Lie group analysis and ordinary differential equations, Chichester, J. Wiley \& Sons, 1999.

[13] Kiselev A.V., On the geometry of the Liouville equation: symmetries, conservation laws, and Bäcklund transformations, Acta Appl. Math., 2002, V.72, 33-49.

[14] Fushchych W.I., Serov N.I., The symmetry and some exact solutions of the nonlinear many-dimensional Liouville, d'Alembert and eikonal equations, J. Phys. A: Math. Gen., 1983, V.16, 3545-3658.

[15] Pucci E., Salvatori M.C., Group properties of a class of semilinear hyperbolic equations, Internat. J. NonLinear Mech., 1986, V.21, N 2, 147-155. 
[16] Liouville J., Sur l'équation aux differences partielles $\partial^{2} \log \lambda / \partial u \partial v \pm \lambda / 2 a^{2}=0$, J. Math. Pure Appl., 1853, V.36, 71-72.

[17] Bateman H., Partial differential equations of mathematical physics, New York, Dover, 1944.

[18] Brito F., Leite M.L., Souza-Neto V., Liouville formula under the viewpoint of minimal surfaces, Comm. Pure Appl. Analysis, 2004, V.3, 41-51.

[19] Chen W., Li C., Classification of solutions of some nonlinear elliptic equations, Duke Math. J., 1991, V.63, $615-622$.

[20] Crowdy D.G., General solutions to the 2D Liouville equation, Int. J. Engn. Sci., 1997, V.35, N 2, $141-149$.

[21] Anderson R.L., Ibragimov N.H., Lie-Bäcklund transformations in applications, Philadelphia, SIAM, 1979.

[22] Khater A.H., Callebaut D.K., El-Kalaawy O.H., Bäcklund transformations and exact solutions for a nonlinear elliptic equation modelling isothermal magnetostatic atmosphere, IMA J. Appl. Math., 2000, V. 65, N 1, 97-108.

[23] Champagne B., Hereman W., Winternitz P., The computer calculation of Lie point symmetries of large systems of differential equations, Comput. Phys. Comm., 1991, V.66, 319-340.

[24] Hereman W., Review of symbolic software for Lie symmetry analysis, Math. Comp. Modelling, 1997, V.25, 115-132 (see also [6, Vol. 3, Chapter 13]).

[25] Baumann G., Symmetry analysis of differential equations with Mathematica, Berlin, Springer, 2000.

[26] Samokhin A.V., Nonlinear M.H.D. equations: symmetries, solutions and conservation laws, Dokl. Akad. Nauk. SSSR, 1985, V.285, N 5, 1101-1106 (English transl.: Sov. Phys. Dokl., 1985, V.30, N 12, 1020-1022).

[27] Bluman G.W., Cole J.D., The general similarity solution of the heat equation, J. Math. Mech., 1969, V.18, 1025-1042.

[28] Bluman G.W., Cole J.D., Similarity methods for differential equations, Berlin, Springer, 1974.

[29] Fushchych W.I., Tsyfra I.M., On a reduction and solutions of the nonlinear wave equations with broken symmetry, J. Phys. A: Math. Gen., 1987, V.20, L45-L48.

[30] Levi D., Winternitz P., Non-classical symmetry reduction: example of the Boussinesq equation, J. Phys. A: Math. Gen., 1989, V.22, 2915-2924.

[31] Winternitz P., Lie groups and solutions of nonlinear partial differential equations, in Integrable Systems, Quantum Groups, and Quantum Field Theories, Proc. XXIII GIFT Intern. Seminar (1992, Salamanca), Editors L.A. Ibort and M.A. Rodríguez, NATO ASI Ser. C, V.409, Dordrecht, Kluwer, 1993, 429-495.

[32] Fushchych W.I. (Editor), Symmetry analysis of equations of mathematical physics, Kyiv, Institute of Mathematics of Acad. Sci. of Ukraine, 1992.

Fushchych W.I., Conditional symmetries of the equations of mathematical physics, in Modern Group Analysis: Advanced Analytical and Computational Methods in Mathematical Physics, Proc. Intern. Workshop (1992, Acireale), Editors N.H. Ibragimov, M. Torrisi and A. Valenti, Dordrecht, Kluwer, 1993, 231-239.

[33] Cicogna G., Gaeta G., Partial Lie-point symmetries of differential equations, J. Phys. A: Math. Gen., 2001, V.34, N 3, 491-512.

[34] Cicogna G., Partial symmetries and symmetric sets of solutions to PDE's, in Symmetry and Perturbation Theory, Proc. 2002 SPT Conference (2002, Cala Gonone), Editors S. Abenda, G. Gaeta and S. Walcher, Singapore, World Scientific, 2002, 26-33.

Cicogna G., Symmetric sets of solutions to differential problems, in Proceedinds of Fourth International Conference "Symmetry in Nonlinear Mathematical Physics" (July 9-15, 2003, Kyiv), Editors A.G. Nikitin, V.M. Boyko and R.O. Popovych, Proceedings of Institute of Mathematics, Kyiv, 2004, V.43, Part 1, 120-127.

[35] Olver P.J., Rosenau Ph., The construction of special solutions to partial differential equations, Phys. Lett. A, 1986, V.114, N 3, 107-112.

Olver P.J., Rosenau Ph., Group-invariant solutions of differential equations, SIAM J. Appl. Math., 1987, V.47, N 2, 263-278.

[36] Olver P.J., Symmetry and explicit solutions of partial differential equations, Appl. Num. Math., 1992, V.10, 307-324.

[37] Pucci E., Similarity reductions of partial differential equations, J. Phys. A: Math. Gen., 1992, V.25, 26312640.

[38] Pucci E., Saccomandi G., On the weak symmetry group of partial differential equations, J. Math. Anal. Appl., 1992, V.163, N 2, 588-598.

Pucci E., Saccomandi G., Evolution equations, invariant surface conditions and functional separation of variables, Phys. D, 2000, V.139, 28-47. 
[39] Olver P.J., Direct reduction and differential constraints, Proc. Roy. Soc. London Ser. A, 1994, V.444, 509523.

[40] Zhdanov R.Z., Tsyfra I.M., Reduction of differential equations and conditional symmetries, Ukr. Math. J., 1996, V.48, 595-602.

[41] Popovych R.O., On reduction and $Q$-conditional (nonclassical) symmetry, in Proceedings of the Second International Conference "Symmetry in Nonlinear Mathematical Physics" (July 7-13, 1997, Kyiv), Editors M.I. Shkil', A.G. Nikitin and V.M. Boyko, Kyiv, Institute of Mathematics, 1997, V.2, 437-443; mathph/0207015.

[42] Zhdanov R.Z., Tsyfra I.M., Popovych R.O., Precise definition of reduction of partial diferential equations, J. Math. Anal. Appl., 1999, V.238, 101-123; math-ph/0207023.

[43] Yehorchenko I.A., Differential invariants and construction of conditionally invariant equations, math$\mathrm{ph} / 0304029$.

[44] Cicogna G., 'Weak' symmetries and adapted variables for differential equations, Int. J. Geom. Meth. Mod. Phys., 2004, V.1, N 1-2, 23-31.

Cicogna G., A discussion on the different notions of symmetry of differential equations, in Proceedinds of Fifth International Conference "Symmetry in Nonlinear Mathematical Physics" (June 23-29, 2003, Kyiv), Editors A.G. Nikitin, V.M. Boyko, R.O. Popovych and I.A. Yehorchenko, Proceedings of Institute of Mathematics, Kyiv, 2004, V.50, Part 1, 77-84.

[45] Cicogna G., Laino M., On the notion of conditional symmetry of differential equations, Rev. Math. Phys., 2006, to appear.

[46] Grasso D., Califano F., Pegoraro F., Porcelli F., Phase mixing and island saturation in Hamiltonian reconnection, Phys. Rev. Lett., 2001, V.86, N 22, 5051-5054.

[47] Del Sarto D., Califano F., Pegoraro F., Secondary instabilities and vortex formation in collisionless fluid megnetic reconnection, Phys. Rev. Lett., V.91, 2003, N 23, 235001-1/4.

[48] Kadomtsev B.B., Pogutse O.P., Theory of electron transport in a strong magnetic field, Soviet Phys. JETP Lett., 1984, V.39, N 5, 269-272.

[49] Bogoyavlenskij O.I., Infinite symmetries of the ideal MHD equilibrium equations, Phys. Lett. A, 2001, V.291, 256-264.

[50] Nucci M.C., Group analysis for M.H.D. equations, Atti Sem. Mat. Fis. Univ. Modena, 1984, V.33, N 1, $21-34$.

[51] Nikitin A.G., Popovych R.O., Group classification of nonlinear Schrödinger equations, Ukr. Math. J., 2001, V.53, N 8, 1255-1265; math-ph./0301009.

[52] Popovych R.O., Yehorchenko I.A., Group classification of generalised eikonal equations, Ukr. Math. J., 2001, V.53, N 11, 1841-1850; math-ph/0112055.

[53] Popovych R.O., Ivanova N.M., New results on group classification of nonlinear diffusion-convection equations, J. Phys. A: Math. Gen., 2004, V.37, 7547-7565; math-ph/0306035.

[54] Güngör F., Lahno V.I., Zhdanov R.Z., Symmetry classification of KdV-type nonlinear evolution equations, J. Math. Phys., 2004, V.45, 2280-2313; nlin.SI/0201063.

[55] Zhdanov R.Z., Lahno V.I., Group classification of the general evolution equation: local and quasilocal symmetries, SIGMA, 2005, V.1, paper 009, 7 pages; nlin.SI/0510003.

[56] Wesson J., Tokamaks, 2nd ed., The Oxford Engineering Series, V.48, Oxford, Clarendon, 1997.

[57] Cicogna G., "Symmetry classification" of a class of PDE's containing several arbitrary functions, in preparation.

[58] Lovelace R.V.E., Mehanian C., Mobarry C.M., Sulkanen M.E., Theory of axisymmetric magnetohydrodynamic flows-disks, Astrophys. J. Suppl., 1986, V.62, 1-37.

[59] Euler N., Steeb W.-H., Mulser P., Symmetries of a nonlinear equation in plasma physics, J. Phys. A: Math. Gen., 1991, V.24, L785-L787.

[60] Kovalev V.F., Krivenko S.V., Pustovalov V.V., Symmetry group of Vlasov-Maxwell equations in plasma theory, J. Nonlinear Math. Phys., 1996, V.3, N 1-2, 175-180.

[61] Kovalev V.F., Bychenkov V.Yu., Tikhonchuk V.T., Particle dynamics during adiabatic expansion of a plasma bunch, J. Exp. Theor. Phys., 2002, V.95, N 2, 226-241.

[62] Kovalev V.F., Bychenkov V.Yu., Analytic solutions to Vlasov equations for expanding plasmas, Phys. Rev. Lett., 2003, V.90, N 18, 185004-1/4. 